\section{The extraordinary}

\section{properties of magnetic oxides}

\author{
B. Raveau and A. Maignan \\ Laboratoire CRISMAT, CNRS/ENSICAEN, 6 boulevard du \\ Maréchal Juin, 14050 CAEN cedex 4 - France
}

$T$ he physical properties of transition metal oxides have been currently the object of many investigations, since the discovery in 1986, of superconductivity at high temperature in cuprates. In these chemically complex materials, the electronic configuration of the transition elements, varies with its valency and is susceptible to adopt various spin states. As a result, strongly correlated electron interactions are generated, leading to complex magnetic and metal-insulator transitions. In this respect, magnetic oxides, containing transition elements such as manganese, cobalt or ruthenium exhibit most fascinating properties.

One of the most famous classes of magnetic oxides concerns the manganites with the perovskite structure (Fig. 1a), which exhibit colossal magnetoresistance (CMR) properties. In these oxides of generic formula $\mathrm{Ln}_{1-\mathrm{x}} \mathrm{A}_{\mathbf{x}} \mathrm{MnO}_{3}(\mathrm{~A}=\mathrm{Ca}, \mathrm{Sr}, \mathrm{Ba})$, double exchange phenomena take place, which require a hopping of charge carriers from a $\mathrm{Mn}^{3+}$ to a $\mathrm{Mn}^{4+}$ ion through an oxygen atom. This effect generates a ferromagnetic metallic state at low temperature, and a metal-insulator transition versus temperature, which coincides with a ferromagnetic to paramagnetic (or antiferromagnetic) transition (Fig. 1b). The latter are also coupled with a structural transition, implying the disappearance of the static Jahn-Teller distortion due to $\mathrm{Mn}^{3+}$, in the ferromagnetic state. As a consequence, at the vicinity of the Curie Temperature $\mathrm{T}_{\mathrm{C}}$ a large magnetoresistance can be obtained: the resistance of these materials can be decreased by several orders of magnitude by an external magnetic field of some teslas. A part of these manganites, those which contain smaller $\mathrm{A}$ cation $\left(\mathrm{Ca}^{2+}\right)$, exhibit a more complex physical behavior, due to orbital and charge ordering phenomena. In these oxides, the manganese octahedra are occupied by $\mathrm{Mn}^{3+}$ and $\mathrm{Mn}^{4+}$ species in an ordered way, forming stripes of $\mathrm{Mn}^{3+}$ distorted octahedra due to Jahn-Teller effect, which alternate with stripes of quasi-regular $\mathrm{Mn}^{4+}$ octahedra. The physics of the latter compounds is then governed by the competition between ferromagnetism and charge/orbital ordering, i.e. the orbital-charge ordered state which is antiferromagnetic and insulating transforms to a metallic ferromagnetic state on the application of a sufficiently high magnetic field, leading also to CMR effect. In these materials, orbital-charge ordering can be destroyed by doping the manganese sites with magnetic cations such as chromium or ruthenium, which by magnetic coupling with adjacent manganese induce ferromagnetism and metallicity, so that spectacular modifications of the magnetic phase diagrams of these systems are obtained. In this way, insulator-metal transitions can be induced and CMR effects are enhanced under much lower magnetic fields.

Another interesting property of these manganites deals with their ability to exhibit a spin glass like behavior, for small A site cations in the composition range close to $\mathrm{CaMnO}_{3}(\mathrm{x} \sim 0.8-0.9)$.

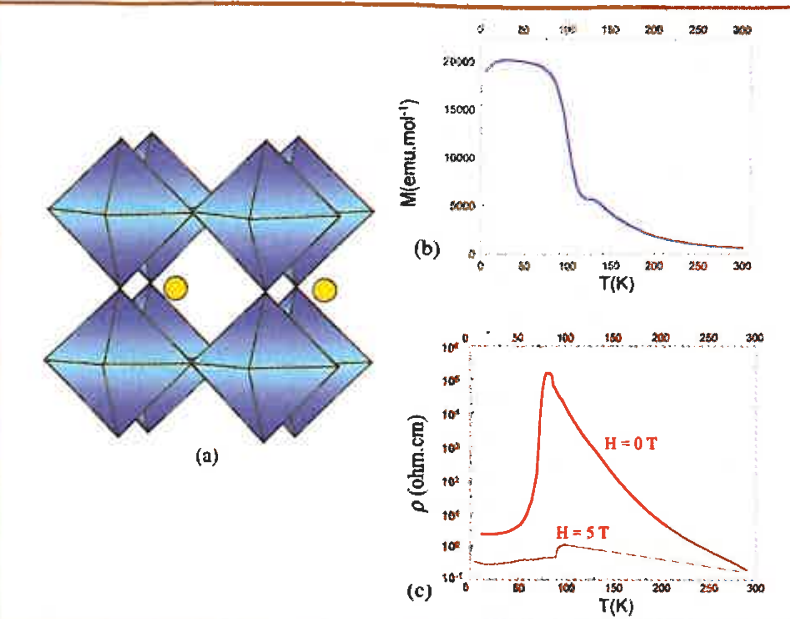

A Fig. 1: The CMR perovskite manganites $L n_{1-x} A_{x} M n O_{3}$ : (a) The perovskite structure built up of corner-sharing $\mathrm{MnO}_{6}$ octahedra forming cages where $\mathrm{Ln}^{3+}$ and $\mathrm{A}^{2+}$ cations are located (b) Ferromagnetic to paramagnetic transition, and (c) corresponding insulator to metal transition ( $\mathrm{H}=0$ zero field) and field effect upon resistivity $(H=5 \mathrm{~T})$ observed in $\operatorname{Pr}_{0.7} \mathrm{Ca}_{0.25} \mathrm{Sr}_{0.05} \mathrm{MnO}_{3}$.

Moreover at the boundary between this region and charge ordered region, CMR properties are also observed.

In fact, the unusual behavior of the manganites is based on a new phenomenon, the electronic phase separation. It has indeed been observed that charge ordered insulating regions coexist at low temperature with small ferromagnetic regions at a submicrometer scale, in a coherent manner in the same matrix. Thus, the CMR effect for the low $\mathrm{T}_{\mathrm{C}}$ manganites results from the percolative conduction through the small ferromagnetic domains embedded in the antiferromagnetic insulating matrix. Such an electronic phase segregation was also predicted from the large magnetostriction effects observed in these materials, and described either as dynamic magnetic polarons, or as static phase segregation. Many other properties of these manganese perovskites are closely relat-

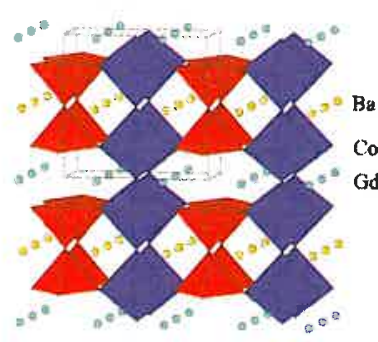

(a)
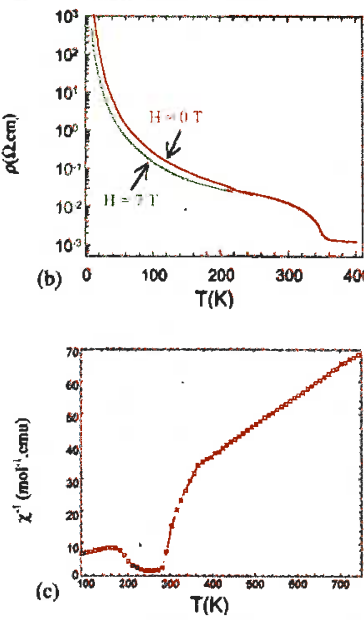

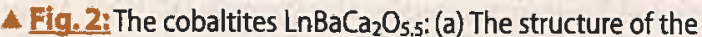
ordered oxygen deficient structure built up from rows of $\mathrm{CoO}_{6}$ octahedra and $\mathrm{COO}_{5}$ pyramids, and involving layers of $\mathrm{Ln}^{3+}$ and $\mathrm{Ba}^{2+}$ cations alternately. (b) The metal to insulator transition of the manganite $\mathrm{GdBaCO}_{2} \mathrm{O}_{5.5}$, (c) The corresponding magnetic transition in the paramagnetic regime. 
ed to the peculiar double exchange phenomena, such as the photo induced metal-insulator transition, as well as the electrical field induced magnetic transition that have been recently observed. The curious effect of thermal cycling upon the resistivity, and the step like behavior at low temperature of their magnetization, resistivity and specific heat versus magnetic field at low temperature, are also certainly related to the phase separation that exists in these compounds, and suggest a martensitic type mechanism.

The high degree of spin polarisation of the conduction electrons in these oxides is of great interest since it can be used to achieve large low field magnetoresistance, which is of capital importance for magnetic recording, or sensing applications. Artificially prepared grain boundaries in thin films allow indeed promising magnetoresistance values to be reached. Finally it must be emphasized that the $3 \mathrm{D}$ perovskites are not the only manganites which exhibit CMR. High magnetoresistance can also be obtained in the 2D Ruddlesden and Popper manganites, such as $\mathrm{Ln}_{2-\mathrm{x}} \mathrm{A}_{1+\mathrm{x}} \mathrm{Mn}_{2} \mathrm{O}_{7}$, but the low dimensionality of these oxides strongly damages their Curie temperatures. In the same manner, the perovskite structure is not the only one to exhibit magnetoresistance. Large magnetoresistance has also been discovered in the pyrochlore $\mathrm{Tl}_{2} \mathrm{Mn}_{2} \mathrm{O}_{7}$ which contains only $\mathrm{Mn}^{4+}$ species suggesting a different origin of CMR.

Cobalt oxides form also a very important family with extraordinary magnetic properties. This is for instance the case of the compounds $\mathrm{LnBaCo}_{2} \mathrm{O}_{5.5}(\mathrm{Ln}=$ lanthanide), which exhibit a metal-insulator (MI) transition coupled with a spin transition. These materials are ordered oxygen deficient perovskites, characterized by a layered ordering of the $\mathrm{Ln}^{3+}$ and $\mathrm{Ba}^{2+}$ cations, so that rows of $\mathrm{CoO}_{6}$ octahedra alternate with rows of $\mathrm{CoO}_{5}$ tetragonal pyramids (Fig. 2a). As a result, there exist two sorts of trivalent cobalt in the structure at high temperature $\left(\mathrm{T}>\mathrm{T}_{\mathrm{MI}}\right)$ : the octahedral cobalt has a high spin (HS) $\mathrm{t}_{2 \mathrm{~g}}{ }^{4} \mathrm{e}_{\mathrm{g}}{ }^{2}$ configuration, whereas the pyramidal one has an intermediate spin (IS) $\mathrm{t}_{2 \mathrm{~g}}{ }^{5} \mathrm{e}_{\mathrm{g}}{ }^{1}$ configuration. In contrast to manganites, the metal to insulator transition appears in these oxides at decreasing temperature (Fig. 2b) around $300-350 \mathrm{~K}$, and coincides with the transition in the paramagnetic susceptibility (Fig. 2c). This strong coupling between magnetism and transport properties is explained by a spin transition of octahedral $\mathrm{Co}^{3+}$ species from high spin (HS) $\mathrm{t}_{2 \mathrm{~g}}{ }^{4} \mathrm{e}_{\mathrm{g}}{ }^{2}$ to low spin (LS) $\mathrm{t}_{2 \mathrm{~g}}{ }^{6}$, the $\mathrm{t}_{2 \mathrm{~g}}{ }^{5} \mathrm{e}_{\mathrm{g}}{ }^{1}$ (IS) pyramidal cobalt being unchanged at the transition. Recent thermoelectric power studies of these oxides, corroborate this viewpoint showing that $S$ changes of sign at the transition from n-type in the metallic state to p-type in the insulating state. Moreover, these cobaltites also exhibit negative giant magnetoresistance at lower temperature. For instance, resistance ratio higher than 10 can be obtained under $7 \mathrm{~T}$ (Fig. 2b),

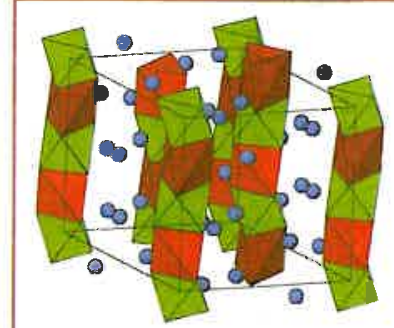

(a)

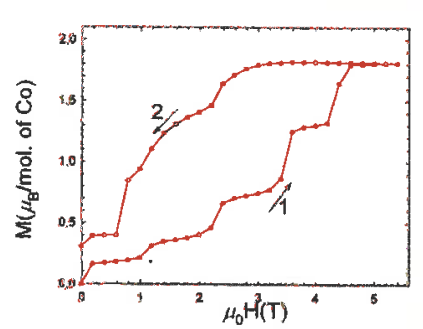

(b)
4 Fig. 3: The cobaltite $\mathrm{Ca}_{3} \mathrm{CO}_{2} \mathrm{O}_{6}$ : (a) The structure consists of $\left[\mathrm{CO}_{2} \mathrm{O}_{6}\right]_{\infty}$ chains of face sharing $\mathrm{CoO}_{6}$ octahedra and $\mathrm{CoO}_{6}$ trigonal prisms forming a triangular array. (b) Magnetization half loop $M(H)$ of this phase registered at $T=2 K$.

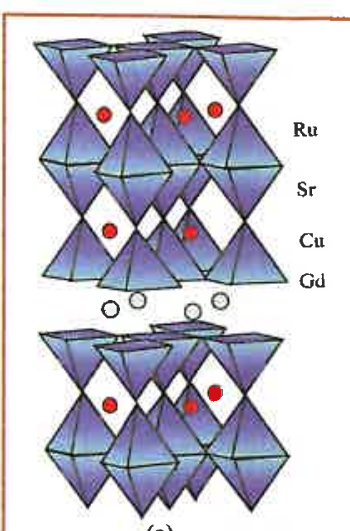

(a)

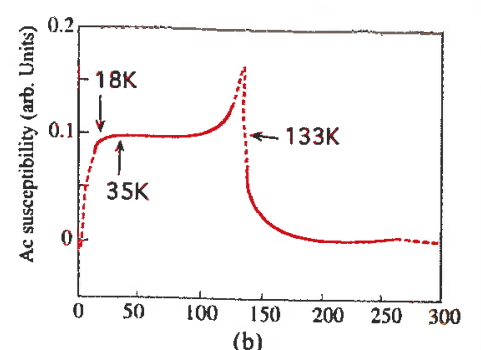

(b)
A Fig. 4: The ferromagnetic superconductor $\mathrm{RuSr}_{2} \mathrm{GdCu}_{2} \mathrm{O}_{8}$ : (a) The structure consists of single layers of $\mathrm{RuO}_{6}$ octahedra sandwiched between layers of $\mathrm{CuO}_{5}$ pyramids, gadolinium and strontium layers are stacked with a 1-2 order (b) The ac susceptibility curve shows that superconductivity is achieved at $18 \mathrm{~K}$ (inset at $35 \mathrm{~K}$ ), whereas ferromagnetism appears below $133 \mathrm{~K}$ according to Chmaissen et al.

in the temperature range $\mathrm{T}<200 \mathrm{~K}$, where a second transition from the paramagnetic to the antiferromagnetic state has been achieved. Note that negative magnetoresistance has also been observed in several other cobaltites: the perovskite $\mathrm{La}_{1-\mathrm{x}} \mathrm{S}_{\mathrm{x}} \mathrm{CoO}_{3}$ and the bismuth based cobaltites, $(\mathrm{Bi}, \mathrm{Cd})_{1} \mathrm{Sr}_{2} \mathrm{CoO}_{5}$ with a layered structure similar to the 1201-type cuprate.

Such a coupling of the magnetic and transport properties in the cobaltites is not limited to the oxygen deficient perovskite structure. A metal-insulator transition correlated with a spin state transition of $\mathrm{Co}^{3+}$ is also observed at room temperature in the cobaltite $\mathrm{TlSr}_{2} \mathrm{CoO}_{5}$. In the latter oxide, whose $2 \mathrm{D}$ structure is an intergrowth of rock salt type layers " $\mathrm{TlSrO}_{2}$ " with single perovskite layers " $\mathrm{SrCoO}_{3}$ ", the metallic state involves strong ferromagnetic interactions, induced by the cooperative spin transition that takes place at lower temperature.

Another example of cobalt oxides is also very attractive for its particular magnetic properties, the one dimensional cobaltite $\mathrm{Ca}_{3} \mathrm{CO}_{2} \mathrm{O}_{6}$. The rhombohedral structure of this phase (Fig. 3a) consists of $\left[\mathrm{Co}_{2} \mathrm{O}_{6}\right]$ chains running along the $c$ axis of the hexagonal cell. In each chain, one $\mathrm{CoO}_{6}$ octahedron alternates with one $\mathrm{CoO}_{6}$ trigonal prism. The great interest of these compounds deals with the fact that a transition from a ferrimagnetic to a ferromagnetic state is induced on application of a magnetic field. A ferromagnetic intrachain coupling exists along $\vec{c}$, whereas an antiferromagnetic intrachain coupling is obtained in the $(a, b)$ plane. Nevertheless the $C_{0}-C o$ intrachain distances are much larger than the $\mathrm{Co}$-Co interchain distances, so that the magnetism of $\mathrm{Ca}_{3} \mathrm{CO}_{2} \mathrm{O}_{6}$ can be described on the basis of a planar Ising triangular lattice where each chain plays the role of one spin. Thus, the magnetic susceptibility of this phase exhibits two transitions versus temperature at $24 \mathrm{~K}$ and $12 \mathrm{~K}$, which correspond to the setting of the interchain antiferromagnetic coupling and spin freezing $\left(\mathrm{T}_{\mathrm{f}}\right)$ respectively. In this Ising triangular ferromagnet, $\mathrm{AC}$ susceptibility measurements show large shift of $T_{\mathrm{f}}$ from 12 to $16.5 \mathrm{~K}$, as the frequency increases by three orders of magnitude. Remarkably, five plateaus can be observed at $2 \mathrm{~K}$ on the $\mathrm{M}(\mathrm{H})$ curve (Fig. 3b), which characteristic magnetic fields are separated by $\sim 1.2 \mathrm{~T}$. This feature is reminiscent of the quantum tunneling of mangnetization encountered in high-spin macromolecules.

Ruthenium oxides exhibit also an extremely rich physics from the magnetism viewpoint. Several members of the Ruddlesden 
and Popper series $\mathrm{Sr}_{n+1} \mathrm{Ru}_{n} \mathrm{O}_{3 n+1}$, with $\mathbf{n}=1,2,3, \infty$ are metallic ferromagnets with $T_{C}$ ranging from $105 \mathrm{~K}$ to $165 \mathrm{~K}$, whereas the $n=1$ member $\mathrm{Sr}_{2} \mathrm{RuO}_{4}$, has often been considered as close to the ferromagnetic order but is in fact a superconductor with a very low critical temperature of $\sim 1 \mathrm{~K}$. Remarkably, the former compounds, show a Fisher-Langer type anomaly of the conductivity versus temperature at $T_{C}$, which is suppressed under a few $T$. In contrast, the calcium homologous phases which are isostructural, exhibit very different properties: the perovskite $\mathrm{CaRuO}_{3}(\mathrm{n}=1)$ is a paramagnetic metal, whereas $\mathrm{Ca}_{2} \mathrm{RuO}_{4}(\mathrm{n}=2)$ is an antiferromagnetic insulator. As a consequence, solid solutions also show complex magnetic properties, as for example the oxides $\mathrm{Ca}_{2-\mathrm{x}} \mathrm{Sr}_{\mathrm{x}} \mathrm{RuO}_{4}$, for which a metal-insulator transition, associated with a structural change and magnetic ordering is observed, for low strontium contents. But the most fascinating properties of ruthenium based oxides have been recently obtained for the ruthenocuprates of the $\mathrm{RuSr}_{2} \mathrm{GdCu}_{2} \mathrm{O}_{8}$ family. The physical behavior of this oxide is unique in that, superconductivity and ferromagnetism coexist within the same matrix. The structure of this phase (Fig. 4a) derives from that of the $92 \mathrm{~K}$-superconductor $\mathrm{YBa}_{2} \mathrm{Cu}_{3} \mathrm{O}_{7}$, by replacing the $\mathrm{CuO}_{4}$ groups by $\mathrm{RuO}_{6}$ octahedra. It can be described as an oxygen deficient perovskite built up of single octahedral perovskite ruthenium layers sandwiched between pyramidal copper layers, layers of $\mathrm{Gd}^{3+}$ and $\mathrm{Sr}^{2+}$ being stacked according to a "1-2" order, between the $\mathrm{CuO}_{2}$ and $\mathrm{RuO}_{2}$ planes. The remarkable coexistence of superconductivity with $a T_{c} \sim 35 \mathrm{~K}$, and of ferromagnetism with a $T_{C} \sim 133 \mathrm{~K}$ is demonstrated from $\mathrm{AC}$ susceptibility measurements (Fig. 4b). Such a behavior shows the important role of the layered architecture of the structure, in order to conciliate these two contradictory properties.

In conclusion, these few examples and those well known on iron oxides show that transition metal oxides represent a vast field of investigation for the discovery of new extraordinary magnetic properties.

\section{References}

\section{For manganites:}

Colossal Magnetoresistance, Charge ordering and Relation Properties of Manganese Oxides. Edit. C.N.R. Rao and B. Raveau. 1998 - World Scientific.

Ibarra et al., Magnetostriction in mixed valent magnetic oxides: in Modern Trends in Magnetostriction Study and Applications. Edit M.R.J. Gibbs - 2000 - Kluwer Acad. Publi.

\section{For cobaltites:}

C. Martin et al., Appl. Phys. Lett. 71, 1421 (1997).

J-C. Burley et al., J. Solid State Chem, accepted 2002.

A.Maignan et al., Eur. Physic. J. B. 15, 657 (2000)

M. Coutanceau et al., Solid State Comm. 96, 569 (1995).

C. Frontera et al., J. Solid State Chem. accepted 2002; Phys. Rev. B 65, 180405 (2002).

M. Respaud et al., Phys. Rev. B, 64, 214401 (2001).

W.S. Kim et al., Solid State Com 116, 609 (2000).

\section{For ruthenates:}

O. Chmaissen et al., Phys. Rev. B 61, 6401 (1999).

S. Malo et al., Internal J. of Inorg. Mat. 2, 601 (2000).

G. Cao et al., Phys. Rev. B 56, R 5740 (1197); ibid 56, 321 (1997).

O. Friedt et al., Phys. Rev. B 63, 174432 (2001). 